\title{
Ageing and hygroscopicity variation of black carbon particles in Beijing measured by a quasi-atmospheric aerosol evolution study (QUALITY) chamber
}

\section{Jianfei Peng et al.}

Correspondence to: Jianfei Peng (pengjianfeipku@gmail.com), Min Hu (minhu@pku.edu.cn) and Renyi Zhang (renyi-zhang @geos.tamu.edu)

The copyright of individual parts of the supplement might differ from the CC BY 3.0 License. 


\section{Instrumentation}

Scanning Mobility Particle Sizer (SMPS). Aerosols were first dried using a Perma Pure Nafion dryer bringing the aerosol to a solid state at a stable RH of $15 \%$ and then directed through an ${ }^{85} \mathrm{Ke}$ charging source to bring aerosols to charge equilibrium. The aerosol flow was passed through a differential mobility analyzer (DMA) and then counted with a condensation particle counter (CPC, 3762, TSI, Inc.). The DMA operated with a sheath flow of $6.5 \mathrm{lpm}$ and a sample flow of $1 \mathrm{lpm}$.

Aerosol particle mass analyzer (APM). The APM (model 3600, Kanomax Inc., Japan) was comprised of two concentric cylindrical electrodes that rotated at the same angular speed on a common axis. The outer cylinder was maintained at ground, while a voltage was applied to the inner cylinder. When particles with a specific mass-to-charge ratio experienced a balance between the electrostatic and centrifugal forces, they penetrated through the APM and were detected by a CPC. By stepping the voltage applied to the APM inner cylinder and passing the aerosol flow through the $\mathrm{CPC}$, the mass distribution of $\mathrm{BC}$ particles was determined.

Humidified Tandem Differential Mobility Analyzer (HTDMA) system. The HTDMA can provide information of particle hygroscopicity by measuring the growth rate in particle diameter at high relative humidity $(\mathrm{RH})$. The system was comprised of two tandem DMAs and one CPC, with a Nafion dryer placed before the first DMA and a Nafion humidifier between the two DMAs. During sampling, a fixed voltage was set to the first DMA to select particles with the same dry diameter $(\mathrm{RH}<30 \%)$. Then, the humidifier increases the RH to approximately $90 \%$ and particles undergo hygroscopic growth. The wet size distribution of particles is then measured by the combination of the second DMA and CPC.

Could condensation nuclei counter (CCNC). The two-column CCNC (CCN-200, DMT, Boulder, CO, USA) was used to measure the ability of BC particles to become $\mathrm{CCN}$ at different supersaturations. A constant supersaturation (SS) in the range between $0.1 \%$ and $0.8 \%$ is established by applying a constant temperature gradient across the chamber. BC particles in the flow will experience this supersaturation at the chamber, and those growing to diameters larger than $1 \mathrm{~mm}$ are counted as CCN at the outlet of the supersaturated column with an optical particle counter (Reberts and Nenes, 2005). In the chamber experiment, column A directly measured the dry $\mathrm{BC}$ sample to obtain total activated $\mathrm{BC}$ particle concentration, while column B connects to the exit of DMA to measure concentration of activated BC particle at the peak size inside the chamber.

The high-resolution time-of-flight aerosol mass spectrometer (HR-ToF-AMS). To obtain the evolution of chemical composition of BC particles during aging process, a HR-ToF-AMS (Aerodyne Research, Inc., USA) was utilized to measure the non-refractory chemical components of BC particles. Ambient aerosol flow entered into an aerodynamic lens through a critical orifice, which focused and accelerated particles into a narrow beam. The particle size was determined by measuring the flight time across a fixed distance. A rotating mechanical chopper defined time zero of particle flight, while the end of particle flight was defined as the time of mass spectrometric detection. At the end of the flight, particles were vaporized 
by impaction on a resistively heated surface $\left(600{ }^{\circ} \mathrm{C}\right)$, ionized by electron impact ionization (EI), and analyzed by a mass spectrometer. The chemical species detected included organic species, nitrate salts, and sulfate salts, whereas crustal material, sea salt, and black carbon could not be detected. Compared with the Q-AMS, the high mass resolution of the HR-ToF-AMS has a greatly improved ability to identify and quantify ions at the same integer $\mathrm{m} / \mathrm{z}$. 
Table S1 Instruments used in this study

\begin{tabular}{|c|c|c|c|}
\hline Instruments & Manufacturer & Species/Parameters & Time resolution \\
\hline HR-TOF-AMS & Aerodyne Research inc. & 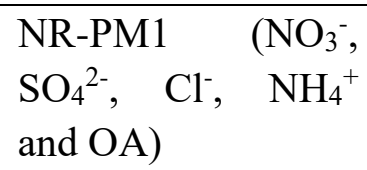 & $5 \mathrm{~min}$ \\
\hline $\begin{array}{l}\text { SMPS } \\
\text { and CPC) }\end{array}$ & TSI & $\begin{array}{l}\text { Particle number size } \\
\text { distribution }\end{array}$ & $5 \mathrm{~min}$ \\
\hline ID-CIMS & Custom built & Sulfuric acid & $1 \mathrm{~min}$ \\
\hline HTDMA & Custom built & $\begin{array}{l}\text { Hygroscopic } \\
\text { growth factor of } \\
\text { particles }\end{array}$ & $5 \mathrm{~min}$ \\
\hline $\mathrm{CCN}$ & DMT Inc., US & $\begin{array}{l}\text { Cloud condensation } \\
\text { nuclei concentration }\end{array}$ & $10 \mathrm{~min}$ \\
\hline APM & KANOMAX Inc. & Particle mass & $10 \mathrm{~min}$ \\
\hline $\begin{array}{l}\text { Trace level gas } \\
\text { analyzer }\end{array}$ & Thermo Inc. & $\mathrm{CO}, \mathrm{O}_{3}, \mathrm{NOx}, \mathrm{SO}_{2}$ & $1 \mathrm{~min}$ \\
\hline TEOM & Thermo Inc. & $\begin{array}{l}\text { PM2.5 } \\
\text { concentration }\end{array}$ & $1 \mathrm{~min}$ \\
\hline
\end{tabular}




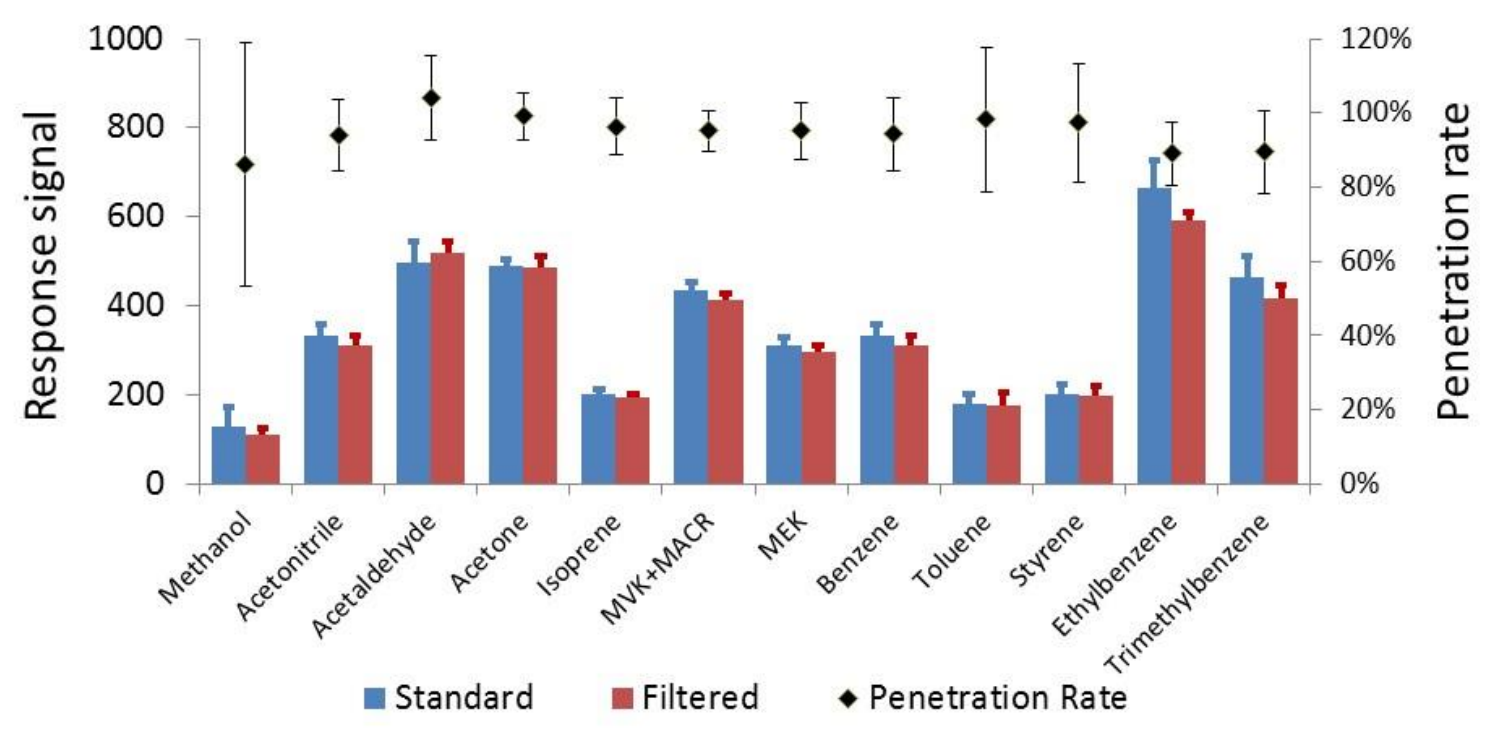

Figure S1. Penetration rate of several volatile organic components (VOCs) through the e-PTFE membrane in the QUALITY chamber. The blue and red bars represent the signal of VOCs before and after passing through the e-PTFE membrane, respectively, and the black diamonds represent the penetration rate. The error bars correspond to one standard deviation. The concentration was 3 ppbv for all VOC species. 


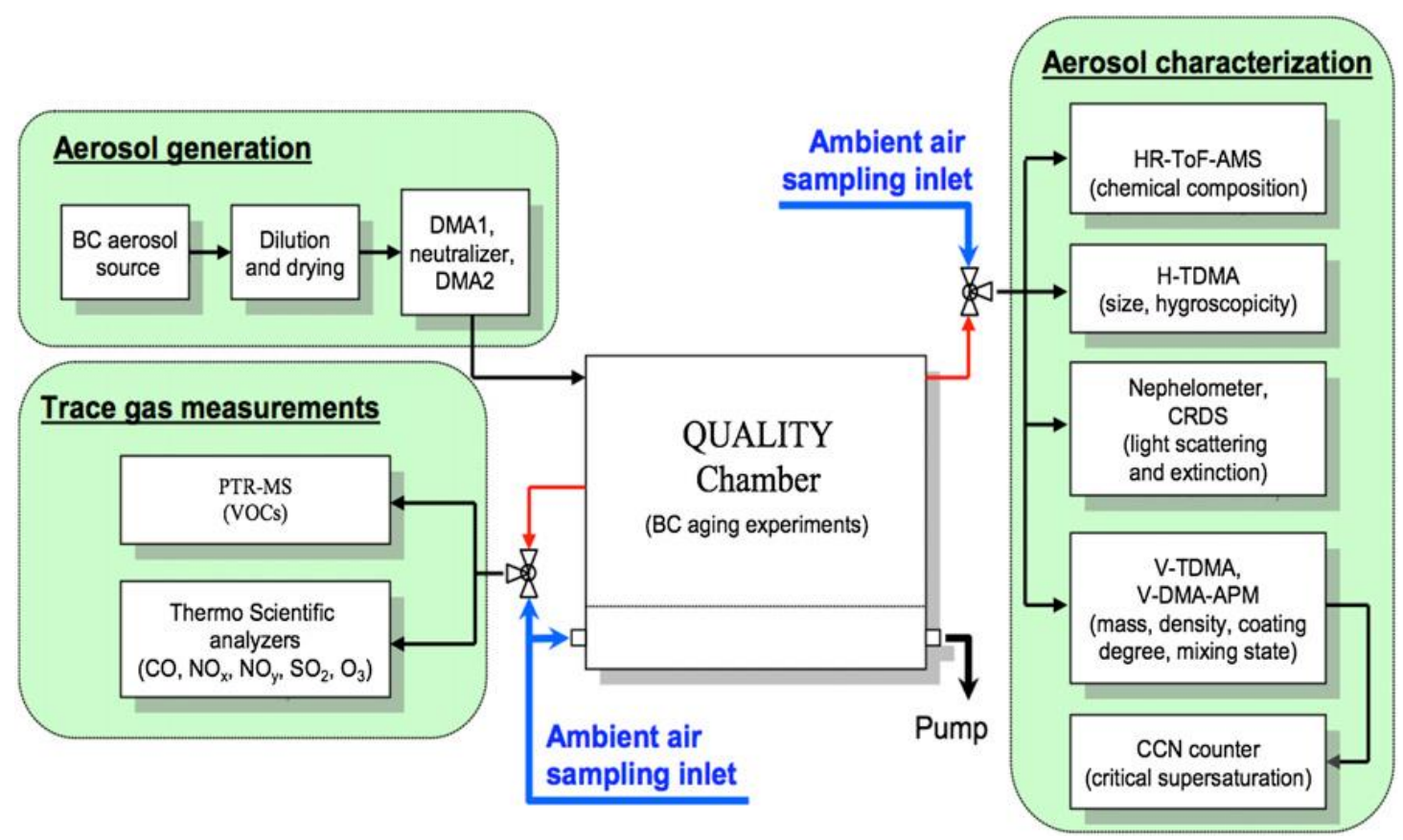

Figure S2. Schematic depiction of the injection and measurement system of the QUALITY chamber experiments. A series of instruments were used to measure the BC particle properties, including a Scanning Mobility Particle Sizer to measure size distribution, a Differential Mobility Analyzer-APM (DMA-APM) system to measure the effective density, a high-resolution time-of-flight AMS (HR-ToF-AMS) to measure the chemical composition, a cloud condensation counter ( $\mathrm{CCN})$ to measure the hygroscopicity, and a photoacoustic extinctionmeter (PAX) to measure the optical properties. TDMA, tandem differential mobility analyzer; PTRMS, proton transfer reaction-mass spectrometry. In order to decrease the loss of particles to the chamber walls due to natural static charge, the monodisperse $\mathrm{BC}$ particles passed through an X-ray neutralizer. A blower was utilized after the X-ray neutralizer to introduce monodisperse $\mathrm{BC}$ particles to the chamber through a port at the side of the reaction chamber. 

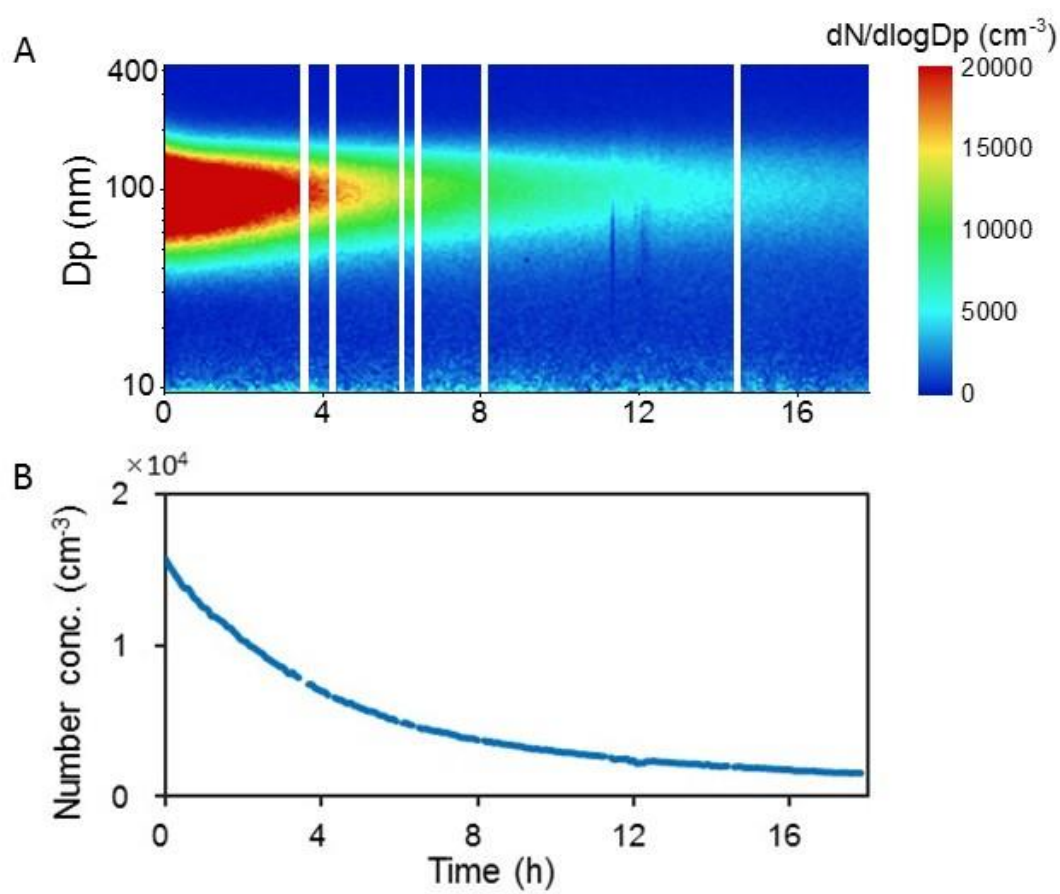

Figure S3. Wall loss of particles in QUALITY chamber. (A) size distribution of particles as a function of time; (B) decay of total particle number concentration 

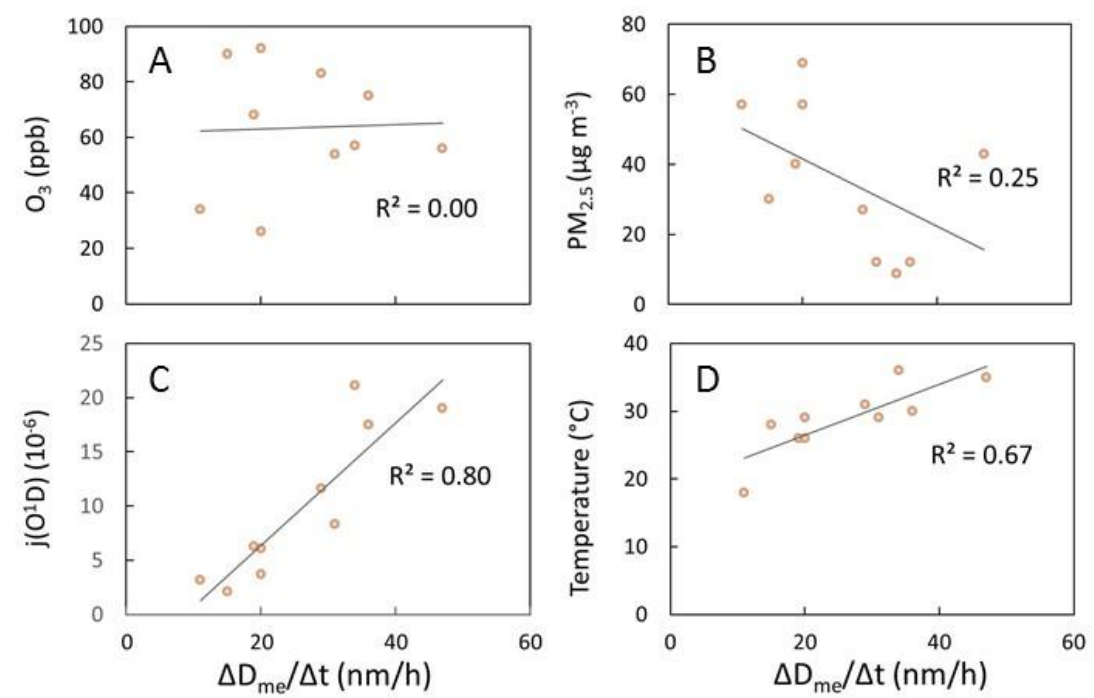

Figure S4. Correlation of the growth rate of the mass equivalent diameter $\left(\Delta D_{m e} / \Delta t\right)$ with $\mathrm{O}_{3}$ (A), $\mathrm{PM}_{2.5}$ (B) concentration, $\mathrm{J}\left(\mathrm{O}^{1} \mathrm{D}\right)(\mathrm{C})$, and temperature (D) in Beijing. Each point represents the average value for each experiment. 